\title{
"Managing Child-to-Parent (reverse) relationship instead of Parent-to-Child during execution of a transaction in Oracle"
}

\author{
Mrs. Shefali Trushit Naik \\ (Sr. Lecturer, H.L. Institute of Computer Applications, Ahmedabad University)
}

\begin{abstract}
This article represents how to use deferred constraint in Oracle to manage child-parent relationship instead of parent-child relationship.

Keywords: - Child-parent relationship, Constraint, Database, Database Management System, Oracle, Parentchild relationship, Relationship
\end{abstract}

Most of the computer professionals, academicians and students know the traditional way of handling one to many relationship using parent and child table. Whenever, there is a one-to-many relationship in a database, we used to define a primary or composite key in the parent table and it is referred as a foreign key in child table. These primary and foreign key constraints are defined at the time of creation of parent and child tables. Once the tables are created successfully with the specific constraints, first we insert the record in a parent table and then the related record is entered into child table. If we try to enter child table first, then database software gives an error of violation of referential integrity rule. This method of handling parent-child relationship is acceptable worldwide, but it is appropriate for the tables where all the fields in the parent record are independent from the fields of child records.

For ex., If the parent table is an EVENT table with fields (Event_id, Event_name) and the child table is EVENT_PARTICIPATION table with fields (Event_id, Participant_id, Participant_name). Event_id is a primary key of EVENT table which is a foreign key in EVENT_PARTICIPATION table. In this case, there are many participants for one event. Before record is inserted into EVENT_PARTICIPATION table, the related event should be entered in EVENT table. For this case, it is acceptable.

Now, consider other case where there is a parent table INTERNAL_MARKS with fields (Std_no, Class_code, Internal_marks, Internal_percentage, Internal_status, Internal_grade) and child table EXAM_MARKS with fields (Std_no, Class_code, Exam_id, Subject1_marks, Subject2_marks, Subject3_marks, Subject4_marks, Subject5_marks, Total, Percentage, Status, Grade). The syntax to create both the tables in Oracle DBMS is given below.

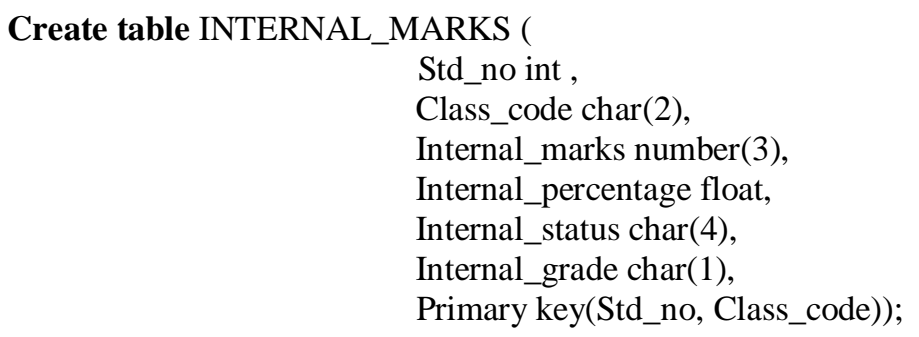

Create table EXAM_MARKS(

Std_no int ,

Class_code char(2),

Exam_id char(5),

Subject1_marks number(2),

Subject2_marks number(2),

Subject3_marks number(2),

Subject4_marks number(2),

Subject5_marks number(2),

Total number(3),

Percentage float,

Status char(4),

Grade char(1), 
Primary key(Std_no, Class_code, Exam_id),

Foreign key(Std_no, Class_code) references

INTERNAL_MARKS (Std_no, Class_code));

During the year, the student appears for three internal exams. At the end of the year, each student's result is processed using some application. In that application, there is a data entry form to enter student's each internal exam's marks and final internal marks are calculated from these marks by applying some formula. This is a single transaction, in which record should be first inserted into INTERNAL_MARKS table as it is a parent table and then related records should be inserted into EXAM_MARKS table which is a child table. But as a business rule, records should be inserted first into child table and then into parent table; which is not possible. Try to execute the following code in oracle.

declare

begin

insert into exam_marks values (1,'fy','exam1', $\quad 25,35,45,37,34,176,70.4$, 'pass','b');

insert into exam_marks values(1,'fy','exam2', 25,35,45,37,34,176,70.4,'pass','b');

insert into exam_marks values(1,'fy','exam3', 25,35,45,37,34,176,70.4,'pass','a');

insert into internal_marks values(1,'fy',206,82.4, 'pass','a');

commit;

end;

The above block in Oracle will display an error message "ORA-02291: integrity constraint (SHEFALI.SYS_C005688) violated - parent key not found" when executed.

As a solution when we write a code, we have to write following SQL statements in Oracle as following to solve the above problem :

declare

begin

Insert into INTERNAL_MARKS values(1,'FY', null, null, null, null)

Insert into EXAM_MARKS values(1,'FY','Exam1', 25, 35, 45, 37, 34, 176, 70.4,'Pass','B');

Insert into EXAM_MARKS values(1,'FY','Exam2', 35, 45, 45, 47, 44, 216, 86.4,'Pass','A');

Insert into EXAM_MARKS values(1,'FY','Exam3', 45, 47, 47, 42, 46, 227, 90.8,'Pass','A');

Update INTERNAL_MARKS set Internal_marks=206, Internal_percentage=82.4, Internal_status='Pass', Internal_grade='A' where Std_no=1 and Class_code='FY';

Commit;

end;

From the above statements, we can say that we need one update statement to change the internal_mark, Internal_percentage, Internal_status and Internal_grade of a student. This is because we can't insert records in EXAM_MARKS until the related record is inserted into INTERNAL_MARKS table. The same transaction could be handled differently by defining the foreign key constraint as "deferred". When we define "deferred" constraint, it is only checked at the commit point of a transaction. The clause "deferrable initially deferred" should be specified when foreign key is defined, to indicate that Oracle should check this constraint at the end of subsequent transactions. Deferred foreign key constraints are not checked until the commit statement is executed. To implement this, the table EXAM_MARKS could be created as following.

Create table EXAM_MARKS (

Std_no int ,

Class_code char(2),

Exam_id char(5),

Subject1_marks number(2),

Subject2_marks number(2),

Subject3_marks number(2),

Subject4_marks number(2),

Subject5_marks number(2),

Total number(3),

Percentage float,

Status char(4), 
Grade char(1),

Primary key(Std_no, Class_code, Exam_id),

Foreign key(Std_no, Class_code) references

INTERNAL_MARKS (Std_no, Class_code)

deferrable initially deferred);

Now, try to execute the following SQL statements which gave error in the above case.

declare

begin

insert into exam_marks values(1,'fy','exam1',25, 35,45,37,34,176,70.4,'pass','b');

insert into exam_marks values(1,'fy','exam2',25,

$35,45,37,34,176,70.4$, 'pass','b');

insert into exam_marks values( 1 ,'fy','exam3',25,

$35,45,37,34,176,70.4$, 'pass','a');

insert into internal_marks values(1,'fy',206,82.4,

'pass','a');

commit;

end;

It will be executed successfully without giving any integrity violation error, though we are inserting child record before inserting the parent record. The only condition is, they must be included in the same transaction and COMMIT should be executed at the end of transaction.

Note: Deferred integrity constraints should only be used when absolutely necessary.

\section{References}

[1] Database Systems: The Complete Book by Hector Garcia, Jeff Ullman, and Jennifer Widom. (http://infolab.stanford.edu/ $\sim$ ullman/dscb.html)

[2] A First Course in Database Systems by Jeff Ullman and Jennifer Widom.

(http://infolab.stanford.edu/ ullman/fcdb.html)

[3] Gradiance SQL Tutorial. (http://www.gradiance.com/STwelcomeAWS.html)

[4] http://www.oracle-base.com/articles/8i/ConstraintCheckingUpdates.php

[5] http://docs.oracle.com/cd/B19306_01/server.102/b14200/clauses002.htm

[6] http://www.java2s.com/Tutorial/Oracle/0120__Table/DeferredConstraints.htm

[7] Deferred Integrity Constraints in Oracle8 by Jeff Hunter, Sr. Database

Administrator(http://www.idevelopment.info/data/Oracle/DBA_tips/Database_Administration/DBA_12.shtml)

\section{About the Author}

Frushit Naik is working as a Senior Lecturer in H.L. Institute of Computer Applications, Ahmedabad ımedabad, Gujarat, India. She has more than twelve years of experience in academics. Her areas of latabases, Software Engineering, Computer System Architecture, Software Project Management and 